\title{
Absence of residual quasiparticle conductivity in the underdoped cuprate $\mathrm{YBa}_{2} \mathrm{Cu}_{4} \mathrm{O}_{8}$
}

\author{
N.E. Hussey \\ Department of Physics, Loughborough University, Loughborough LE11 3TU, U.K. \\ S. Nakamae, K. Behnia \\ UPR 5 CNRS, ESPCI, 10 Rue Vauquelin, 75005 Paris, France. \\ H. Takagi, C. Urano \\ Department of Advanced Materials Science, Graduate School of Frontier Sciences, University of Tokyo, Hongo 7-3-1, Tokyo, \\ 113-8656, Japan. \\ S. Adachi and S. Tajima \\ Superconducting Research Laboratory, International Superconductivity Technology Center, Shinonome 1-10-13, Tokyo 135, \\ Japan.
}

Experimental evidence for $d$-wave superconductivity in high- $T_{c}$ cuprates is now well established. The presence of nodes in the gap is expected to produce a finite density of well-defined quasiparticle (QP) excitations at low energies that dominate the low- $T$ physics. For a pure $d$-wave superconductor with line nodes on the Fermi surface, for example, the density of states (DOS) is linear in the excitation energy, giving rise to a $T^{2}$ dependence of the low- $T$ specific heat and thermal conductivity (assuming a constant scattering rate). This excitation spectrum, however, is altered significantly in the presence of impurities. For an anisotropic two-dimensional (2D) superconductor with scattering in the unitary limit, a band of impurity states is expected to develop whose width $\gamma$ grows with increasing impurity concentration $n_{i m p}$, leading to a finite zero-energy DOS 1 田. Lee showed that the residual conductivity is independent of $n_{i m p}$, the result of compensation between the increased DOS and a reduction in the associated transport lifetime [2]. This residual, or "universal" conductivity develops at low $T$ in the so-called "dirty" limit, $k_{B} T \leq \gamma$. Similarly, the low- $T \kappa(T)$ will be dominated by QP states in the vicinity of the line nodes, and is given by the expression [4]

$$
\kappa_{\text {res }} / T \approx(n / d)\left(k_{B}^{2} / 3\right)\left(v_{F} / v_{2}\right)
$$

where $n / d$ is the stacking density of $\mathrm{CuO}_{2}$ planes and $v_{F}$ and $v_{2}$ are the energy dispersions (QP velocities) perpendicular and tangential to the Fermi surface respectively.
The issue of localization of electronic states in $d$-wave superconductors, however, is a complex problem and many competing viewpoints prevail [2 8]. Balatsky and Salkola [5], for example, argue that the long-range nature of hopping between impurity states along the nodal directions leads to strong overlap of the impurity wave functions along the diagonals of the square lattice, and ultimately to "extended" impurity states. Senthil and coworkers [6, [7], on the other hand, argue that quantum interference effects destabilize the extended QP states and lead to a vanishing DOS at zero energy (See also [8] for a similar conclusion). They coined the term "superconducting insulator" [7] to describe such a superconductor with localized states.

Experimentally, the observation of a finite linear term in $\kappa(T)$ in pure and $\mathrm{Zn}$-doped $\mathrm{YBa}_{2} \mathrm{Cu}_{3} \mathrm{O}_{7-\delta}(\mathrm{Y} 123)$ by Taillefer et al. [9] appeared to confirm the existence of zero-energy quasiparticles. Moreover, the size of this term was indeed found to be "universal", i.e. independent of Zn concentration, in agreement with Lee's prediction. Similar behavior was later reported for $\mathrm{Bi}_{2} \mathrm{Sr}_{2} \mathrm{CaCu}_{2} \mathrm{O}_{8}$ (Bi2212) by Behnia et al., using irradiated crystals [10]. More recently, the magnitude of $\kappa_{\text {res }} / T$ in $\operatorname{Bi} 2212\left(\approx 0.15 \mathrm{~mW} / \mathrm{cm} . \mathrm{K}^{2}\right)$ was shown to be consistent with absolute values of $v_{F} / v_{2}$ estimated from angle-resolved photoemission (ARPES) [11,12].

Despite this apparent consistency between theory and experiment, $\kappa_{\text {res }} / T$ has only been reported for two compounds, both at their optimum doping level, and it is not immediately obvious how $\kappa_{\text {res }} / T$ will vary across the phase diagram. ARPES 11] and penetration depth measurements [13] support claims that $v_{F} / v_{2}$, and thereby $\kappa_{\text {res }} / T$, increase in the underdoped regime. On the other hand, in certain underdoped cuprates, where $T_{c}$ has been suppressed in high magnetic fields [14, 15], there is a marked tendency towards localization below $T_{c}$, suggesting a vanishing QP contribution at low $T$. Clearly, low- $T$ $\kappa(T)$ measurements on underdoped cuprates are important to help clarify this seemingly contradictory behavior. 
With this in mind, we have carried out the first low$T \kappa(T)$ measurements on the underdoped cuprate Y124 $\left(T_{c}=80 \mathrm{~K}\right)$, which is a self-doped, stoichiometric cuprate and therefore relatively free of disorder. Below $0.25 \mathrm{~K}$, $\kappa(T) \approx T^{3}$ for both $a$ - and $b$-axis currents, consistent with a phonon heat conduction in the ballistic regime. The residual linear QP term, however, is either absent or is negligibly small, with an upper bound of 0.02 $\mathrm{mW} / \mathrm{cm} \cdot \mathrm{K}^{2}$. This result reveals that the universal conductivity scenario breaks down dramatically in underdoped Y124. One compelling possibility is that the QP states in Y124 are localized at low $T$, due to the proximity to the superconductor/insulator $(\mathrm{S} / \mathrm{I})$ boundary, and therefore do not contribute to the low- $T$ heat transport.

The Y124 crystals were grown by a flux method described elsewhere 16]. For this particular study, three plate-like crystals were selected, two with their longest dimension along the $b$-axis, the other along the $a$-axis. Approximate dimensions were $0.25 \times 0.16 \times 0.015 \mathrm{~mm}^{3}$ for the $\kappa_{a}$ crystal (labelled hereafter as a $\sharp 1$ ) and $1 \times 0.09$ x $0.05 \mathrm{~mm}^{3}$ and $0.8 \times 0.25 \times 0.06 \mathrm{~mm}^{3}$ for the two $\kappa_{b}$ crystals, $\mathrm{b} \sharp 1$ and $\mathrm{b} \sharp 2$. $T_{c}=80 \mathrm{~K}$ for all crystals, with a transition width, measured resistively, of less than $1 \mathrm{~K}$.

$\kappa(T)$ for each crystal was measured between $0.14 \mathrm{~K}$ and $1 \mathrm{~K}$ using a conventional steady-state four-probe technique that allowed the electrical resistivity $\rho_{a, b}(T)$ of each sample to be measured in situ without changing the contact configuration. Gold wires were attached as electrical contacts using Dupont 6838 silver paint. The $\rho_{a, b}(T)$ behavior was found to be in excellent agreement with previous measurements [17], with room temperature values, $\rho_{a}=350 \mu \Omega \mathrm{cm}$ and $\rho_{b}=90 \mu \Omega \mathrm{cm}$ (for both $b$-axis crystals). This large in-plane anisotropy arises from the high conductivity of carriers on the quasi-1D $\mathrm{CuO}$ chains that run parallel to the $b$-axis (see schematic inset to Figure 1) and confirms not only the high quality of the crystals used in this study, but also that current flow in each case is uniaxial. The temperature gradient was measured by two $\mathrm{RuO}_{2}$ thermometers connected to the "voltage" contacts through the gold wires, and the thermometers were supported by long, thin superconducting $\mathrm{Nb}-\mathrm{Ti}$ wires to minimize heat losses. Uncertainties in the absolute magnitudes of $\kappa(T)(\rho(T))$, mainly due to the finite contact dimensions on these small crystals, are estimated to be around $15 \%$ for $\kappa_{b}\left(\rho_{b}\right)$ and around $25 \%$ for $\kappa_{a}\left(\rho_{a}\right)$.

The $\kappa(T)$ data for all crystals are shown on doublelogarithmic axes in Figure 1. The variation of $\kappa_{b}(T)$ for the two $b$-axis crystals is almost identical over the whole temperature range studied, giving us confidence in the reproducibility of our data. Below $0.25 \mathrm{~K}, \kappa_{a}$ and $\kappa_{b}$ both vary approximately as $T^{3}$, consistent with phonon heat transport in the boundary-scattering limit. Above $0.25 \mathrm{~K}, \kappa_{a}(T)$ deviates more strongly from a $T^{3}$ dependence. The origin of the enhancement of $\kappa_{b}$ over $\kappa_{a}$ above $T=0.25 \mathrm{~K}$ is not understood at present, though we assume it reflects an additional contribution to $\kappa$ from the
$\mathrm{CuO}$ chains; either QP conductivity on the chains develops swiftly above $0.25 \mathrm{~K}$ (note that the $\mathrm{CuO}$ chains, being quasi-1D, may be susceptible to charge ordering at very low $T$ ), or there exists an additional channel for phonon heat propagation 18] along the chains that reduces the effects of phonon scattering beyond the ballistic regime. Further measurements in a magnetic field are envisaged to clarify the origin of this anisotropy.

In order to look for evidence of a linear $\kappa_{\text {res }}$, we have re-plotted the low $T$ data in Figure 2 as $\kappa_{a, b} / T$ versus $T^{2}$ and fitted each data set below $0.25 \mathrm{~K}$ to the expression $\kappa_{a, b}=A T+B T^{3}$ 19]. The coefficients for each fit are $A$ $=0.011,-0.007$ and $0.006( \pm 0.02) \mathrm{mW} / \mathrm{cm} . \mathrm{K}^{2}$ and $B$ $=6.50,7.00$ and $10.67( \pm 0.50) \mathrm{mW} / \mathrm{cm} . \mathrm{K}^{4}$ for $\mathrm{a} \sharp 1, \mathrm{~b} \sharp 1$ and $\mathrm{b} \sharp 2$ respectively.

In the boundary-scattering limit, $\kappa_{p h}$ is given by

$$
\kappa_{p h}=1 / 3 \beta<v_{p h}>l_{0} T^{3}
$$

where $\beta$ is the phonon specific heat coefficient, $\left\langle v_{p h}\right\rangle$ the average acoustic sound velocity and $l_{0}=2 \mathrm{w} / \sqrt{\pi}$ is the maximum phonon mean free path. Here, $w$ represents a mean width of the rectangular-shaped crystal. Taking the dimensions of our crystals and suitable values for $\beta$ $\left(=0.5 \pm 0.1 \mathrm{~mJ} / \mathrm{mol} . \mathrm{K}^{4}\right)$ [20] and $\left\langle v_{p h}>(=5 \pm 1\right.$ x $10^{5} \mathrm{~cm} / \mathrm{s}$ ) 21], we obtain estimates for $\kappa_{p h} / T^{3}=4.1$ $\pm 1.2,5.25 \pm 1.5$ and $9.55 \pm 2.0 \mathrm{~mW} / \mathrm{cm} . \mathrm{K}^{4}$ for $\mathrm{a} \sharp 1$, $\mathrm{b} \sharp 1$ and $\mathrm{b} \sharp 2$ respectively 22 . Given the uncertainties in measuring dimensions and contact distances, we believe these values compare favourably with the experimental values. More importantly, the size of the $T^{3}$ term for the two $b$-axis crystals scales well with $w$ and we conclude that the $T^{3}$ contribution is indeed simply the phonon contribution in the ballistic regime.

The most striking result here is the complete absence (to within our experimental accuracy) of the residual linear term in the low- $T \kappa(T)$ for both chain and plane current directions. It should be emphasized, of course, that a zero linear term in $\kappa_{b}$ also implies a negligible $\kappa_{\text {res }}$ within the planes, meaning we have effectively confirmed the absence of the universal QP term in Y124 in all three samples. Moreover, for there to be any finite zero- $T$ intercept in $\kappa / T$, it would require $\kappa_{p h}(T)$ below $0.15 \mathrm{~K}$ to vary as $T^{3+n}$ with $n>0$, which is simply not physical, given that the lattice heat capacity is strictly cubic below $1 \mathrm{~K}$. Thus, we are confident that the main result of this Letter, namely the absence of $\kappa_{r e s}$ in Y124, is robust. For comparison, we also show in the inset to Figure 2, $\kappa_{a b} / T$ for optimally doped Bi2212 [10] measured with the same experimental set-up [23]. In Bi2212, we can clearly distinguish a finite $\kappa_{\text {res }} / T \approx 0.15 \mathrm{~mW} / \mathrm{cm} . \mathrm{K}^{2}$ (shown by a dotted line), that is an order of magnitude larger than the upper limits for $\kappa_{\text {res }} / T$ in Y124.

Despite the overwhelming case for $d$-wave pairing in high- $T_{c}$ cuprates, there is still limited, direct evidence for a $d_{x^{2}-y^{2}}$ order parameter in Y124. Thus, before discussing our result in terms of nodal QP states, we should 
first examine the possibility that there is a finite gap everywhere on the Fermi surface in Y124. First of all, the orthorhombic distortion in Y124, induced by the chains, introduces some $d+s$ admixture in the gap function. As the $s$-component is increased from zero, the position of the nodal lines are first shifted away from $(\pi, \pi)$, but as the $s$-component becomes comparable with the $d$-component, a nodeless gap may form. Secondly, magnetic impurities are thought to induce a local imaginary component that could also give rise to a fully gapped state and a suppression of low $T$ thermal transport 24. This latter possibility, however, is not supported by specific heat data taken on crystals from similar batches to those studied here, which show no sign of a low $T$ Schottky anomaly arising from such magnetic impurities [20. In addition, power law penetration depths have now been observed down to $2 \mathrm{~K}$ for both the $a$ - and $b$-axes in Y124 25,26, suggesting a simple nodal gap picture is equally applicable to Y124. Of course, we cannot rule out completely the possibility of a finite gap in Y124, but if it does exist, it would have to be vanishingly small. In what follows, therefore, we assume that nodal lines are present in Y124 and turn to consider what might be happening to the low-energy QP states in their vicinity.

From (1), we see that $\kappa_{r e s} / T$ is directly proportional to the ratio $v_{F} / v_{2}$ at the nodal positions, so a negligible $\kappa_{\text {res }} / T$ may indicate a sharp gap feature at the nodes at very low energies, induced either by doping, impurities or structural modifications. However, as noted above, $v_{F} / v_{2}$ is expected to increase as we move into the underdoped regime, and indeed, independent estimates of $v_{F} / v_{2}$ from the slope of the low- $T$ penetration depth 26] in Y124 yield an estimate for $\kappa_{\text {res }} / T$ that is larger than those for both optimally doped Bi2212 and Y123. Moreover, given that band structure estimates for $v_{F}$ are similar for Y123 and Y124 27, even with our upper bound estimate for $\kappa_{\text {res }} / T\left(=0.02 \mathrm{~mW} / \mathrm{cm} . \mathrm{K}^{2}\right)$, we obtain a physically unrealistic value $\left(v_{F} / v_{2} \approx 2\right)$ for the gap slope within the nodes in Y124. It appears unlikely, therefore, that the gap structure can itself explain a value of $\kappa_{r e s} / T$ one order of magnitude lower than in Y123.

Another important consideration is the size of the impurity band, $\gamma$. We recall that in the unitary limit, the universal conductivity regime develops below $k_{B} T \leq \gamma$. Thus, the observation of $\kappa_{\text {res }}$ depends not only on the temperature range of the experiment, but also on the energy scale of the impurity band, imposed by the scattering phase shift. However, taking values for the $b$-axis residual resistivity $\rho_{0}=0.5 \mu \Omega \mathrm{cm}$ [17] and plasma frequency $\omega_{p}=2.5 \mathrm{eV}$ 28], we obtain a lower bound estimate of $\gamma$ in the unitary limit of $14 \mathrm{~K}$, some two orders higher than the base temperature of our measurements. (Unfortunately, similar analysis for $\mathrm{J} / / \mathrm{a}$ cannot be performed due to the difficulties in estimating $\rho_{0}$ from $\rho_{a}(T)$ ). Even in the opposite (Born) limit, where $\gamma$ becomes exponentially small and therefore, may fall below our measurement range, the product of the DOS and the lifetime is also energy independent above $k_{B} T \geq \gamma$. Hence, $\kappa_{\text {res }} / T$ should still be constant and finite and the same arguments still apply.

Given these simple yet rather compelling arguments, we are left to consider how QP localization might account for the absence of $\kappa_{\text {res }} / T$ in underdoped Y124. As mentioned above, high magnetic field measurements on the underdoped cuprates $\mathrm{La}_{2-x} \mathrm{Sr}_{x} \mathrm{CuO}_{4}$ [14] and $\mathrm{Pr}_{2-x} \mathrm{Ce}_{x} \mathrm{CuO}_{4}$ [15] revealed that $\rho_{a b}(T)$, though "metallic" at high $T$, tends towards localization as $T \rightarrow 0 \mathrm{~K}$. The origin of this localization phenomenon is unknown at present. However, if the field-induced destruction of superconductivity leads directly to an insulating phase, then it is not unreasonable to assume that this transition is from a superconductor with already localized QP excitations [7]. The crossover from metallic to insulating behavior in the normal state (i.e. above $H_{c 2}$ ) as we approach the Mott insulator, suggests an increasing role of long-range interactions on the mobility of low-energy quasiparticles. The superfluid condensate, suppressed in the vicinity of an impurity, becomes ineffective in screening completely the Coulomb repulsion between quasiparticles in the bound state [29]. As we approach the parent insulator, we expect such interactions to grow, leading to insulating behavior of the quasiparticles above the superconducting condensate and a negligible QP contribution to the low- $T$ heat transport [30]. Such localization in a nominally clean superconductor is an exciting prospect, and measurements on Zn-doped or irradiated Y124 are envisaged to investigate this possibility further. We note here that most impurity models for $d$-wave (cuprate) superconductivity fail to take into account the developing role of long-range interactions between quasiparticles as the S/I boundary is approached. We hope, therefore, that this work stimulates renewed theoretical efforts to understand the nature of QP excitations in the $\mathrm{CuO}_{2}$ planes, deep inside the superconducting state, and in particular in stoichiometric crystals on the underdoped side of the phase diagram.

In conclusion, we have measured the low- $T \kappa(T)$ of stoichiometric, underdoped Y124 and have found that, in marked contrast to optimally doped Y123 and Bi2212, the "universal" QP conductivity term is absent. We have considered several interpretations of this intriguing result, including localization of the quasiparticles themselves, due to enhanced long-range interactions as we approach the S/I boundary. Prior to this work, the observation of the universal conductivity in Y123 and Bi2212 had been widely regarded as solid support for the picture of long-lived quasiparticles above the superconducting ground state of high- $T_{c}$ cuprates. Our surprising result offers important and timely counter evidence that the residual conductivity term is non-universal, and we hope it encourages further debate and investigation into this critical, and still controversial, issue. 
We acknowledge enlightening discussions with A.S. Alexandrov, J.F. Annett, A.V. Balatsky, D.M. Broun, J.R. Cooper, P.J. Hirschfeld, F.V. Kusmartsev, A.P. Mackenzie, A.J. Schofield, L. Taillefer, I. Vekhter and V.W. Wittorff. S.N. acknowledges support from the National Science Foundation under Grant No.INT-9901436. This work was also partly supported by CREST, a Grant in Aid for Scientific Research from the Ministry of Education, Science and Culture, Japan and New Energy and Industrial Technology Department Organization (NEDO).

[1] L.P. Gorkov and P.A. Kalugin, Pis'ma Zh. Eksp. Teor. Fiz. 41, 208 (1985) [JETP Lett. 41, 253 (1985)].

[2] P.A. Lee, Phys. Rev. Lett 71, 1887 (1993).

[3] P. J. Hirschfeld and W. O. Putikka, Phys. Rev. B 50, 10250 (1994)

[4] M.J. Graf et al., Phys. Rev. B 53, 15147 (1996).

[5] A.V. Balatsky and M.I. Salkola, Phys. Rev. Lett. 76, 2386 (1996).

[6] T. Senthil et al., Phys. Rev. Lett. 81, 4704 (1998); T. Senthil and M. P. A. Fisher, preprint, cond-mat/9810238.

[7] S. Vishveshwara, T. Senthil and M. P. A. Fisher, Phys. Rev. B. 61, 6966 (2000).

[8] A.A. Nersesyan, A.M. Tsvelik and F. Wenger, Phys. Rev. Lett. 72, 2628 (1994).

[9] L.Taillefer et al., Phys. Rev. Lett. 79, 483 (1997).

[10] K. Behnia et al., J. Low. Temp. Phys. 117, 1089 (1999).

[11] J. Mesot et al., Phys. Rev. Lett. 83, 840 (1999).

[12] May Chiao et al., preprint, condmat/9910367.

[13] C. Panagopoulos and T. Xiang, Phys. Rev. Lett. 81, 2336 (1998).

[14] Y. Ando et al., Phys. Rev. Lett. 75, 4662 (1995).

[15] P. Fournier et al., Phys. Rev. Lett. 81, 4720 (1998).

[16] S. Adachi et al., Physica 301C, 123 (1998).

[17] N.E. Hussey et al., Phys. Rev. B 56, R11423 (1997).

[18] J.L. Cohn and J. Karpinski, Phys. Rev. B 58, 14617 (1998).

[19] In previous measurements on Y123 and Bi2212, the asymptotic $T^{3}$ regime was observed only at the lower end of the temperature range of our experiments, i.e. below $150 \mathrm{mK}$. The range for boundary-scattering, however, is determined by the sample dimensions, as well as the finite $T$ excitations which might act to scatter the phonons (see P.D. Thacher, Phys. Rev. 156, 975 (1967)). Y124 crystals are appreciably smaller in cross-section than the Y123 or Bi2212 crystals studied previously, and therefore, it is natural to expect the $T^{3}$ behaviour to set in at a correspondingly higher $T$. Moreover, the use of a higher $T$ fit on optimally-doped systems leads to an overestimation, rather than a reduction or vanishing of the linear term.

[20] M. Nohara, private communication.

[21] Wu Ting et al., Phys. Rev. B. 47, 12197 (1993).

[22] For a very thin sample, such as our $a$-axis crystal, the effective width is slightly modified (A.S. Alexandrov, pri- vate communication). However, for the geometry of the $a$-axis crystal used here, this correction is a minor one.

[23] Similar values are found for Y123 12].

[24] R. Movshovich et al., Phys. Rev. Lett. 80, 1968 (1998).

[25] C. Panagopoulos, J.L. Tallon and T. Xiang, Phys. Rev. B 59, R6635 (1999).

[26] D.M. Broun et al., (unpublished).

[27] S. Massidda et al., Physica 176C, 159 (1991).

[28] D.N. Basov et al., Phys. Rev. Lett. 74, 598 (1995).

[29] A.V. Balatsky, M.I. Salkola and A. Rosengren, Phys. Rev. B 51, 15547 (1995).

[30] In the course of preparing this manuscript, we became aware of new low- $T \kappa(T)$ measurements on $\mathrm{Pr}_{2-x} \mathrm{Ce}_{x} \mathrm{CuO}_{4}$ that also show a vanishing $\kappa_{\text {res }} / T$ (L. Taillefer, private communication).

\section{Figure Captures}

Fig.1. $\kappa_{a}(T)$ and $\kappa_{b}(T)$ of Y124, plotted on doublelogarithmic axes. The dashed line represents the $T^{3}$ dependence expected for phonon heat transport in the ballistic regime. The inset shows a simple schematic of the crystal structure of Y124.

Fig.2. $\kappa / T$ versus $T^{2}$ below $0.4 \mathrm{~K}$ for a $\sharp 1$ (closed circles), $\mathrm{b} \sharp 1$ (open circles) and $b \sharp 2$ (open squares). Fits to the expression $\kappa=A T+B T^{3}$ below $0.25 \mathrm{~K}$ are indicated by dashed lines for $\mathrm{b} \sharp 1$ and $\mathrm{b} \sharp 2$ and by a solid line for $a \sharp 1$. The dotted line represents the universal conductivity limit for Bi2212. Inset: Comparison of $\kappa_{a} / T$ for Y124 (closed circles) and $\kappa_{a b} / T$ of Bi2212 [10] (open diamonds), measured in the same apparatus. 


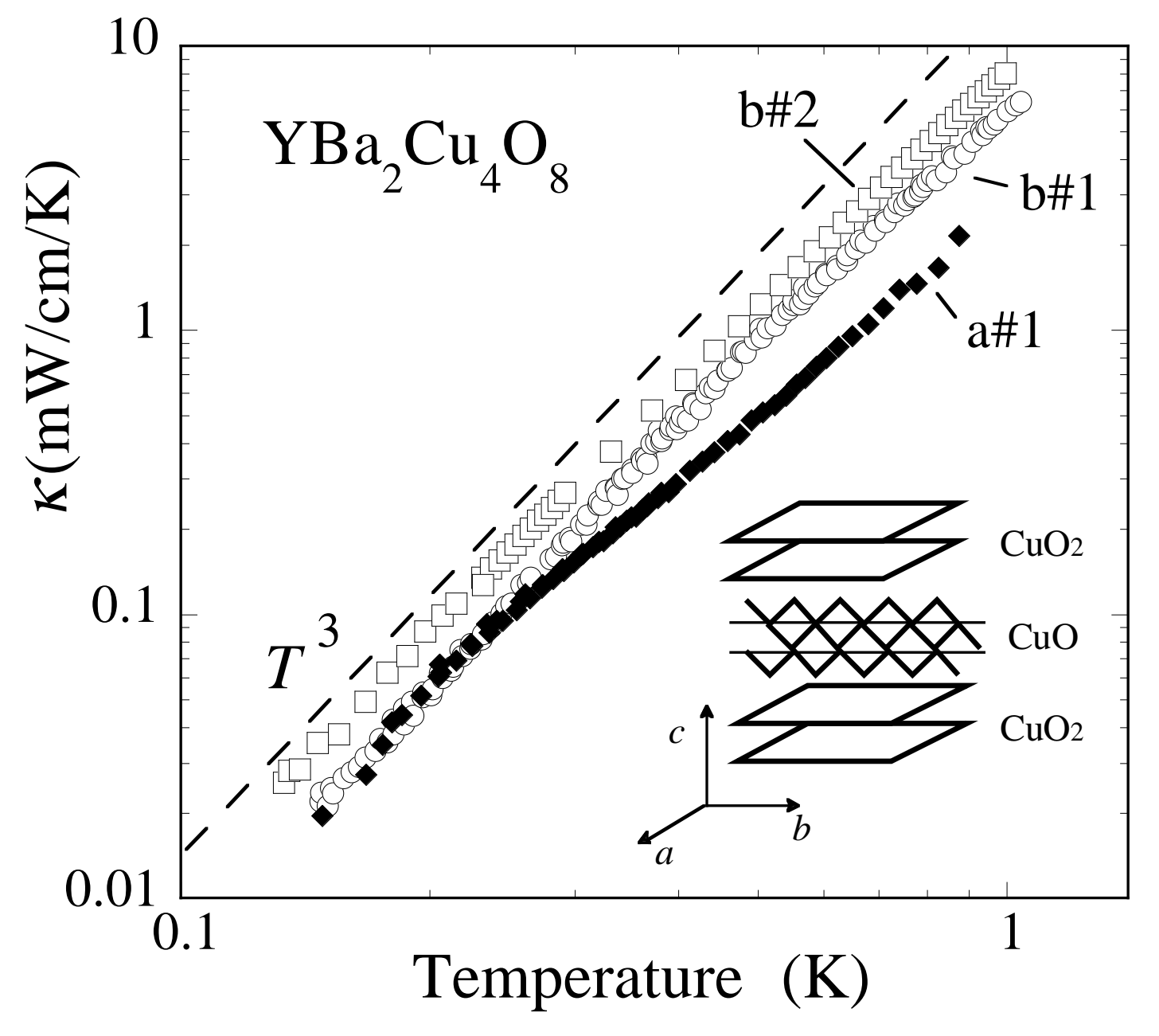

Figure 1

N.E. Hussey et al. 


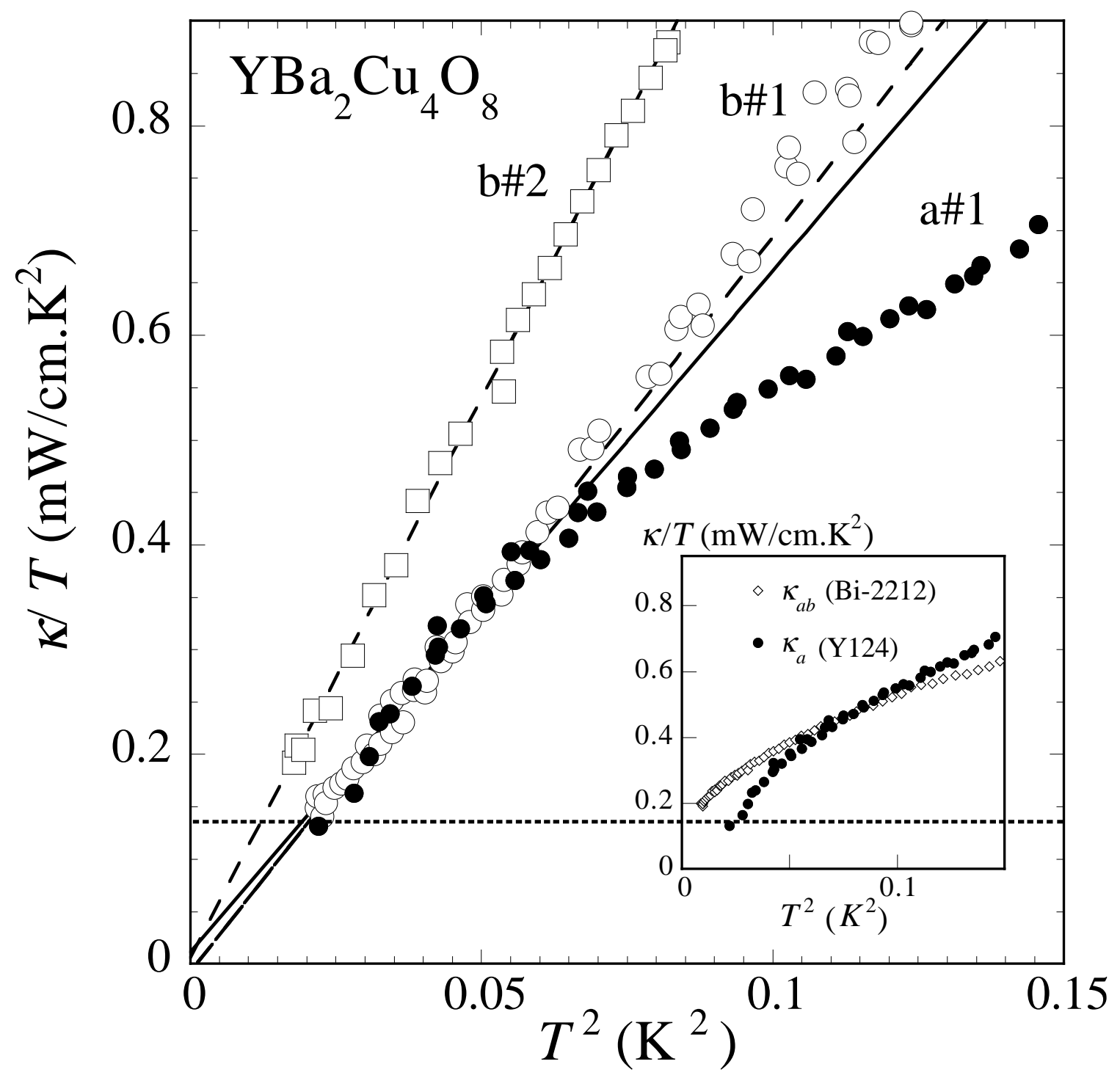

Figure 2

N.E. Hussey et al. 